\title{
ASYMPTOTIC DECAY OF OSCILLATORY SOLUTIONS OF SECOND ORDER DIFFERENTIAL EQUATIONS WITH FORCING TERM
}

\author{
TAKAŜI KUSANO AND HIROSHI ONOSE
}

\begin{abstract}
The ordinary differential equation $\left(p(t) y^{\prime}\right)^{\prime}+q(t) f(y)=r(t)$ and its companion functional differential equation are considered. Sufficient conditions are given which ensure that all oscillatory solutions tend to zero as $t \rightarrow \infty$.
\end{abstract}

1. Introduction. This paper is concerned with the asymptotic behavior of oscillatory solutions of the second order ordinary differential equation

$$
\left(p(t) y^{\prime}(t)\right)^{\prime}+q(t) f(y(t))=r(t)
$$

and the associated functional differential equation

$$
\left(p(t) y^{\prime}(t)\right)^{\prime}+q(t) f(y(g(t)))=r(t),
$$

where $p, q, r, g:[a, \infty) \rightarrow R$ and $f: R \rightarrow R$ are assumed to be continuous. In addition, it will be assumed throughout that $p(t)>0, f(y)$ is nondecreasing, $y f(y)>0$ for $y \neq 0$ and $\lim _{t \rightarrow \infty} g(t)=\infty$.

We shall restrict our attention to solutions $y(t)$ of (A) and (B) which exist on some ray $\left[T_{y}, \infty\right)$ and satisfy $\sup \{|y(t)|: t \geqslant T\}>0$ for every $T \geqslant T_{y}$. Such a solution is said to be oscillatory if the set of its zeros is not bounded; otherwise, it is said to be nonoscillatory.

During the last two decades there has been a great deal of interest in the oscillation theory of differential equations, and many results have appeared in the literature. It seems to us, however, that very little is known about the asymptotic behavior of oscillatory solutions. For equation (A), see the papers of Graef and Spikes [1], [2], Hatvani [3], and Heidel [4]; for equation (B), see the recent work of Singh [5].

The main purpose of this paper is to present some new results regarding this subject. We establish conditions which guarantee that all oscillatory solutions or all bounded oscillatory solutions of (A) and (B) converge to zero as $t \rightarrow \infty$. Thus, in particular, we are able to improve and extend all the results of Singh [5] for equation (B). Our technique is much simpler than the one used by Singh.

Received by the editors June 23, 1976 and, in revised form, November 29, 1976.

AMS (MOS) subject classifications (1970). Primary 34C10, 34K15.

Key words and phrases. Differential equation with forcing term, oscillatory solution, asymptotic behavior.

- American Mathematical Society 1977 
2. Oscillatory solutions of (A). We start with the ordinary differential equation (A). The following notation will be used in the sequel: $q^{+}(t)=$ $\max [q(t), 0]$,

$$
P(t)=\int_{a}^{t} \frac{d s}{p(s)}, \quad \pi(t)=\int_{t}^{\infty} \frac{d s}{p(s)} .
$$

THEOREM 1. Assume that either

(1) $\int_{a}^{\infty} \frac{d t}{p(t)}=\infty, \quad \int_{a}^{\infty} P(t) q^{+}(t) d t<\infty, \quad \int_{a}^{\infty} P(t)|r(t)| d t<\infty$,

or

$$
\int_{a}^{\infty} \frac{d t}{p(t)}<\infty, \quad \int_{a}^{\infty} q^{+}(t) d t<\infty, \quad \int_{a}^{\infty}|r(t)| d t<\infty .
$$

Then all bounded oscillatory solutions of (A) tend to zero as $t \rightarrow \infty$.

Proof. Suppose to the contrary that (A) has a bounded oscillatory solution $y(t)$ such that $\lim \sup _{t \rightarrow \infty}|y(t)|>0$. Then there exist a constant $\delta>0$ and two sequences $\left\{\sigma_{n}\right\},\left\{\tau_{n}\right\}$ of zeros of $y(t)$ with the following properties: $\sigma_{n}<\tau_{n}, \lim _{n \rightarrow \infty} \sigma_{n}=\lim _{n \rightarrow \infty} \tau_{n}=\infty$, and either

$$
y(t)>0 \text { on }\left(\sigma_{n}, \tau_{n}\right), \quad M_{n}=\max _{\left[\sigma_{n}, \tau_{n}\right]} y(t)>\delta, \quad n=1,2, \ldots,
$$

or

$$
y(t)<0 \text { on }\left(\sigma_{n}, \tau_{n}\right), \quad m_{n}=\min _{\left[\sigma_{n}, \tau_{n}\right]} y(t)<-\delta, \quad n=1,2, \ldots
$$

Suppose (3) holds. Let $\left\{t_{n}\right\}$ be a sequence such that $t_{n} \in\left(\sigma_{n}, \tau_{n}\right)$ and $y\left(t_{n}\right)=M_{n}$. Integrating (A) from $t \in\left[\sigma_{n}, t_{n}\right]$ to $t_{n}$ and noting that $y^{\prime}\left(t_{n}\right)=0$, we obtain

$$
\begin{aligned}
p(t) y^{\prime}(t) & =\int_{t}^{t_{n}} q(s) f(y(s)) d s-\int_{t}^{t_{n}} r(s) d s \\
& \leqslant \int_{t}^{t_{n}} q^{+}(s) f(y(s)) d s+\int_{t}^{t_{n}}|r(s)| d s .
\end{aligned}
$$

Dividing (5) by $p(t)$ and integrating over $\left[\sigma_{n}, t_{n}\right]$, we get

(6) $y\left(t_{n}\right) \leqslant \int_{\sigma_{n}}^{t_{n}} \frac{1}{p(t)} \int_{t}^{t_{n}} q^{+}(s) f(y(s)) d s d t+\int_{\sigma_{n}}^{t_{n}} \frac{1}{p(t)} \int_{t}^{t_{n}}|r(s)| d s d t$.

Observing that (1) or (2) implies

(7) $\int_{a}^{\infty} \frac{1}{p(t)} \int_{t}^{\infty} q^{+}(s) d s d t<\infty, \quad \int_{a}^{\infty} \frac{1}{p(t)} \int_{t}^{\infty}|r(s)| d s d t<\infty$,

we deduce from (6) that

(8) $M_{n} \leqslant f\left(M_{n}\right) \int_{\sigma_{n}}^{\infty} \frac{1}{p(t)} \int_{t}^{\infty} q^{+}(s) d s d t+\int_{\sigma_{n}}^{\infty} \frac{1}{p(t)} \int_{t}^{\infty}|r(s)| d s d t$.

It follows therefore that 
(9) $\delta \leqslant f(M) \int_{\sigma_{n}}^{\infty} \frac{1}{p(t)} \int_{t}^{\infty} q^{+}(s) d s d t+\int_{\sigma_{n}}^{\infty} \frac{1}{p(t)} \int_{t}^{\infty}|r(s)| d s d t$,

where $M=\sup y(t)$. In view of (7) the right-hand side of (9) can be made arbitrarily small by letting $n \rightarrow \infty$. This, however, is a contradiction.

If (4) holds, then by a parallel argument we are led to the same type of contradiction. This completes the proof.

THEOREM 2. In addition to the hypotheses of Theorem 1 assume that

$$
\limsup _{|y| \rightarrow \infty} \frac{f(y)}{y}<\infty \text {. }
$$

Then all oscillatory solutions of (A) tend to zero as $t \rightarrow \infty$.

Proof. Let $y(t)$ be an oscillatory solution of (A). We shall show that it is bounded. Suppose the contrary. Then, either $\lim \sup _{t \rightarrow \infty} y(t)=\infty$ or $\lim _{\inf _{t \rightarrow \infty}} y(t)=-\infty$. It suffices to examine the first possibility. We can take sequences $\left\{\sigma_{n}\right\},\left\{\tau_{n}\right\}$ of zeros of $y(t)$ with the properties that $\sigma_{n}<$ $\tau_{n}, \lim _{n \rightarrow \infty} \sigma_{n}=\lim _{n \rightarrow \infty} \tau_{n}=\infty, y(t)>0$ on $\left(\sigma_{n}, \tau_{n}\right)$ and $M_{n}=\max _{\left[\sigma_{n}, \tau_{n}\right]} y(t)$ increases to infinity as $n \rightarrow \infty$. Let $t_{n} \in\left(\sigma_{n}, \tau_{n}\right)$ be such that $y\left(t_{n}\right)=M_{n}, n=$ $1,2, \ldots$ Then, proceeding as in the proof of Theorem 1, we arrive at inequality (8). From this we obtain

$$
1 \leqslant \frac{f\left(M_{n}\right)}{M_{n}} \int_{\sigma_{n}}^{\infty} \frac{1}{p(t)} \int_{t}^{\infty} q^{+}(s) d s d t+\frac{1}{M_{1}} \int_{\sigma_{n}}^{\infty} \frac{1}{p(t)} \int_{t}^{\infty}|r(s)| d s d t .
$$

Since $\left\{f\left(M_{n}\right) / M_{n}\right\}$ is bounded above by (10), letting $n \rightarrow \infty$ in (11), we have a contradiction. It follows that $y(t)$ must be bounded, and, hence, by Theorem 1 , it decays to zero as $t \rightarrow \infty$. The proof is complete.

THEOREM 3. Let the following conditions hold: $q(t) \geqslant 0$,

$$
\int_{a}^{\infty} \frac{d t}{p(t)}<\infty, \quad \int_{a}^{\infty} q(t) d t<\infty, \quad \int_{a}^{\infty}|r(t)| d t<\infty,
$$

and $\left[\pi^{\alpha}(t) p(t) q(t)\right]^{\prime} \leqslant 0$ for some constant $\alpha, 0 \leqslant \alpha<2$.

Then, all oscillatory solutions of (A) approach zero as $t \rightarrow \infty$.

Proof. It suffices to show that every oscillatory solution of (A) is bounded. Actually it can be shown that every solution is bounded. Let $y(t)$ be a solution of (A) defined on $[T, \infty)$. Define

$$
V(t)=\pi^{\alpha}(t)\left(p(t) y^{\prime}(t)\right)^{2}+2 \pi^{\alpha}(t) p(t) q(t) \int_{0}^{y(t)} f(u) d u
$$

We easily see that

$$
\begin{aligned}
V^{\prime}(t) & \leqslant 2 \pi^{\alpha}(t) p(t) r(t) y^{\prime}(t) \leqslant \pi^{\alpha}(t)|r(t)|\left[\left(p(t) y^{\prime}(t)\right)^{2}+1\right] \\
& \leqslant \pi^{\alpha}(t)|r(t)|+|r(t)| V(t),
\end{aligned}
$$

and, hence, 


$$
V(t) \leqslant K+\int_{T}^{t}|r(s)| V(s) d s, \quad t \geqslant T,
$$

where $K=V(T)+\int_{T}^{\infty} \pi^{\alpha}(t)|r(t)| d t$, which is finite since $|r(t)|$ is integrable and $\pi(t) \rightarrow 0$ as $t \rightarrow \infty$. An application of Gronwall's inequality shows that $V(t)$ is bounded, so that there is a constant $k>0$ such that

$$
\left|\pi^{\alpha / 2}(t) p(t) y^{\prime}(t)\right| \leqslant k \text { for } t \geqslant T \text {. }
$$

Dividing the above inequality by $\pi^{\alpha / 2}(t) p(t)$ and integrating, we obtain

$$
|y(t)| \leqslant|y(T)|+\frac{2 k}{2-\alpha}[\pi(T)]^{(2-\alpha) / 2}, \quad t \geqslant T .
$$

It follows that $y(t)$ is bounded. This completes the proof.

EXAMPLE 1. Consider the equation

$$
\begin{aligned}
y^{\prime \prime}(t)+t^{-3} y^{1 / 3}(t)= & t^{-3}[\cos (\log t)+3 \sin (\log t)] \\
& +t^{-10 / 3} \cos ^{1 / 3}(\log t) .
\end{aligned}
$$

The hypotheses of Theorem 2 are satisfied, and so all oscillatory solutions of this equation tend to zero as $t \rightarrow \infty$. One such solution is $y(t)=t^{-1} \cos (\log t)$.

EXAMPLE 2. Consider the equation

$$
\begin{aligned}
& \left(t^{2} y^{\prime}(t)\right)^{\prime}+t^{-3 / 2} y^{3}(t)=t^{-2}[\sin (\log t)-3 \cos (\log t)] \\
& +t^{-15 / 2} \sin ^{3}(\log t)
\end{aligned}
$$

which has a solution $y(t)=t^{-2} \sin (\log t)$. From Theorem 1 it follows that all bounded oscillatory solutions of this equation approach zero as $t \rightarrow \infty$. However, applying Theorem 3, we have a stronger conclusion that all oscillatory solutions tend to zero as $t \rightarrow \infty$.

3. Oscillatory solutions of (B). Let us now consider the functional differential equation (B). In general, the hypotheses of Theorem 1 do not guarantee that all bounded oscillatory solutions of (B) decay to zero as $t \rightarrow \infty$. For example, the equations

$$
y^{\prime \prime}(t)-y(t+\pi)=0, \quad y^{\prime \prime}(t)-y(t-\pi)=0
$$

possess bounded oscillatory solutions $\cos t, \sin t$ that do not damp to zero as $t \rightarrow \infty$. However, using the technique developed in the preceding section, we can prove oscillation theorems closely akin to Theorems 1 and 2 .

THEOREM 4. Assume that either

$$
\int_{a}^{\infty} \frac{d t}{p(t)}=\infty, \quad \int_{a}^{\infty} P(t)|q(t)| d t<\infty, \quad \int_{a}^{\infty} P(t)|r(t)| d t<\infty
$$

or

$$
\int_{a}^{\infty} \frac{d t}{p(t)}<\infty, \quad \int_{a}^{\infty}|q(t)| d t<\infty, \quad \int_{a}^{\infty}|r(t)| d t<\infty .
$$

Then all bounded oscillatory solutions of (B) tend to zero as $t \rightarrow \infty$.

Proof. Let $y(t)$ be a bounded oscillatory solution of (B) such that 
$\lim \sup _{t \rightarrow \infty}|y(t)|>2 \delta$ for some $\delta>0$. Then there are two sequences $\left\{\sigma_{n}\right\},\left\{\tau_{n}\right\}$ of zeros of $y(t)$ with the following properties:

$$
\begin{gathered}
\delta_{n}<\tau_{n}, \quad \lim _{n \rightarrow \infty} \sigma_{n}=\lim _{n \rightarrow \infty} \tau_{n}=\infty, \\
|y(t)|>0 \text { on }\left(\sigma_{n}, \tau_{n}\right) \quad \text { and } \quad M_{n}=\max _{\left[\sigma_{n}, \tau_{n}\right]}|y(t)|>\delta, \quad n=1,2, \ldots
\end{gathered}
$$

Take $t_{n} \in\left(\sigma_{n}, \tau_{n}\right)$ so that $\left|y\left(t_{n}\right)\right|=M_{n}, n=1,2, \ldots$ Upon integrating (B) from $t \in\left[\sigma_{n}, t_{n}\right]$ to $t_{n}$, we find

$$
\left|p(t) y^{\prime}(t)\right| \leqslant \int_{t}^{t_{n}}|q(s)||f(y(g(s)))| d s+\int_{t}^{t_{n}}|r(s)| d s
$$

We divide the above inequality by $p(t)$ and integrate over $\left[\sigma_{n}, t_{n}\right]$ to get

$$
\begin{aligned}
\left|y\left(t_{n}\right)\right| \leqslant & \left|\int_{\sigma_{n}}^{t_{n}} \frac{1}{p(t)} \int_{t}^{t_{n}}\right| q(s)|| f(y(g(s))) \mid d s d t \\
& +\int_{\sigma_{n}}^{t_{n}} \frac{1}{p(t)} \int_{t}^{t_{n}}|r(s)| d s d t .
\end{aligned}
$$

It follows that

$$
M_{n} \leqslant f(M) \int_{\sigma_{n}}^{\infty} \frac{1}{p(t)} \int_{t}^{\infty}|q(s)| d s d t+\int_{\sigma_{n}}^{\infty} \frac{1}{p(t)} \int_{t}^{\infty}|r(s)| d s d t
$$

where $M=\sup |y(t)|$. By virtue of (13) or (14) the right-hand side of (16) tends to zero as $t \rightarrow \infty$. But this is a contradiction, and the proof is complete.

THEOREM 5. In addition to the hypotheses of Theorem 4 assume that (10) holds and

$$
g(t) \leqslant t, \quad \lim _{t \rightarrow \infty} g(t)=\infty .
$$

Then all oscillatory solutions of (B) tend to zero as $t \rightarrow \infty$.

Proof. Let $y(t)$ be an oscillatory solution of (B) defined on $[T, \infty)$. Suppose that $y(t)$ is unbounded: $\lim \sup _{t \rightarrow \infty}|y(t)|=\infty$. We can select two sequences $\left\{\sigma_{n}\right\},\left\{\tau_{n}\right\}$ of zeros of $y(t)$ with the following properties:

$$
\begin{gathered}
\sigma_{n}<\tau_{n}, \quad \lim _{n \rightarrow \infty} \sigma_{n}=\lim _{n \rightarrow \infty} \tau_{n}=\infty, \quad|y(t)|>0 \text { on }\left(\sigma_{n}, \tau_{n}\right), \\
M_{n}=\max _{\left[T, \tau_{n}\right]}|y(t)|=\max _{\left[\sigma_{n}, \tau_{n}\right]}|y(t)|, \quad n=1,2, \ldots,
\end{gathered}
$$

and $M_{n}$ tends increasingly to infinity as $n \rightarrow \infty$. Let $\left\{t_{n}\right\}$ be such that $t_{n} \in\left(\sigma_{n}, \tau_{n}\right)$ and $\left|y\left(t_{n}\right)\right|=M_{n}$. Following the argument in the proof of Theorem 4 , we conclude that inequality (15) holds. Since $g(t) \leqslant t$, we have $|y(g(t))| \leqslant M_{n}$ for $t \leqslant t_{n}$. Therefore it follows from (15) that

(18) $1 \leqslant \frac{f\left(M_{n}\right)}{M_{n}} \int_{\sigma_{n}}^{\infty} \frac{1}{p(t)} \int_{t}^{\infty}|q(s)| d s d t+\frac{1}{M_{1}} \int_{\sigma_{n}}^{\infty} \frac{1}{p(t)} \int_{t}^{\infty}|r(s)| d s d t$,

which leads us to a contradiction in the limit as $n \rightarrow \infty$. Thus $y(t)$ must be bounded, and the assertion follows from Theorem 4 . 
REMARK. Theorem 5 includes the main results of Singh [5, Theorems 1, 2] as a special case.

EXAMPLE 3. Consider the equation with advanced argument

$$
\left(t^{2} y^{\prime}(t)\right)^{\prime}-t^{-2} y^{3}\left(e^{t}\right)=t^{-2}[\sin (\log t)-3 \cos (\log t)]-t^{-2} e^{-6 t} \sin ^{3} t
$$

Theorem 4 implies that all bounded oscillatory solutions of this equation tend to zero as $t \rightarrow \infty$. In fact, $y(t)=t^{-2} \sin (\log t)$ is a solution that satisfies the conclusion of Theorem 4 .

EXAMPLE 4. Consider the equation with delayed argument

$$
\begin{aligned}
& \left(e^{-t} y^{\prime}(t)\right)^{\prime}+e^{-2 t} \cos (\log t) y(\log t) \\
& \quad=e^{-2 t}[\cos t+3 \sin t]+t^{-1} e^{-2 t} \cos ^{2}(\log t)
\end{aligned}
$$

which has a solution $y(t)=e^{-t} \cos t$. By Theorem 5 all other oscillatory solutions of this equation converge to zero as $t \rightarrow \infty$.

We conclude with a result which is an extension of Theorem 4 of Singh [5].

TheOREM 6. Assume that (10) and (17) hold and that

$$
\int_{a}^{\infty}|q(t)| d t<\infty, \quad \int_{a}^{\infty}|r(t)| d t<\infty
$$

and

$$
\underset{t \rightarrow \infty}{\limsup } \int_{t}^{t+c} \frac{d s}{p(s)}<\infty \text { for any } c>0 .
$$

Let $y(t)$ be an oscillatory solution of (B) such that the distances between its consecutive zeros remain bounded. Then $y(t) \rightarrow 0$ as $t \rightarrow \infty$.

Proof. We shall first show that $y(t)$ is bounded. Suppose to the contrary that $\lim \sup _{t \rightarrow \infty}|y(t)|=\infty$. Define $\left\{\sigma_{n}\right\},\left\{\tau_{n}\right\},\left\{t_{n}\right\}$ and $\left\{M_{n}\right\}$ exactly as in the proof of Theorem 5 . We then arrive at inequality (15). Taking (19) into account, we obtain from (15) that

$$
\begin{aligned}
1 \leqslant & \frac{f\left(M_{n}\right)}{M_{n}} \int_{\sigma_{n}}^{\tau_{n}} \frac{d s}{p(s)} \cdot \int_{\sigma_{n}}^{\infty}|q(s)| d s \\
& +\frac{1}{M_{1}} \int_{\sigma_{n}}^{\tau_{n}} \frac{d s}{p(s)} \cdot \int_{\sigma_{n}}^{\infty}|r(s)| d s .
\end{aligned}
$$

Using (10), (19), (20) and the fact that $\left\{\tau_{n}-\sigma_{n}\right\}$ is bounded, we see that a contradiction arises if we let $n \rightarrow \infty$ in (21). This shows that $y(t)$ is bounded.

Next, we shall prove that $y(t) \rightarrow 0$ as $t \rightarrow \infty$. Suppose the contrary. Since no confusion may occur, we again use the same symbols $\left\{\sigma_{n}\right\},\left\{\tau_{n}\right\},\left\{t_{n}\right\},\left\{M_{n}\right\}$ to denote the ones employed in the proof of Theorem 4. Again we obtain inequality (15), and from this we readily deduce that

$$
\begin{aligned}
M_{n} \leqslant & f(M) \int_{\sigma_{n}}^{\tau_{n}} \frac{d s}{p(s)} \cdot \int_{\sigma_{n}}^{\infty}|q(s)| d s \\
& +\int_{\sigma_{n}}^{\tau_{n}} \frac{d s}{p(s)} \cdot \int_{\sigma_{n}}^{\infty}|r(s)| d s,
\end{aligned}
$$


where $M=\sup |y(t)|$. On account of (19), (20) and the boundedness of $\left\{\tau_{n}-\sigma_{n}\right\}$ the right-hand side of (22) tends to zero as $n \rightarrow \infty$. But this is a contradiction, since $\left\{M_{n}\right\}$ is bounded away from zero by a positive constant. This completes the proof.

EXAMPLE 5. Consider the equation

$$
\left(t^{-2} y^{\prime}(t)\right)^{\prime}+t^{-2} y(t-2 \pi)=2 t^{-3} \sin t
$$

which has an oscillatory solution $y(t)=\cos t$. Although the distances of consecutive zeros of cos $t$ remain bounded, it does not converge to zero as $t \rightarrow \infty$. Clearly, (19) is satisfied, but (20) is violated.

ACKNowledgement. The authors would like to express their gratitude to the referee for a number of helpful suggestions.

\section{REFERENCES}

1. J. R. Graef and P. W. Spikes, Continuability, boundedness and asymptotic behavior of solutions of $x^{\prime \prime}+q(t) f(x)=r(t)$, Ann. Mat. Pura Appl. (4) 101 (1974), 307-320. MR 50 \# 13719.

2. Asymptotic behavior of solutions of a second order nonlinear differential equation, $\mathrm{J}$. Differential Equations 17 (1975), 461-476. MR 50 \# 13720.

3. L. Hatvani, On the asymptotic behaviour of the solutions of $\left(p(t) x^{\prime}\right)^{\prime}+q(t) f(x)=0$, Publ. Math. Debrecen 19 (1972), 225-237. MR 48 \#409.

4. J. W. Heidel, A nonoscillation theorem for a nonlinear second order differential equation, Proc. Amer. Math. Soc. 22 (1969), 485-488. MR 40 \# 1648.

5. B. Singh, Asymptotically vanishing oscillatory trajectories in second order retarded equations, SIAM J. Math. Anal. 7 (1976), 37-44.

Department of Mathematics, Faculty of Science, Hiroshima University, Hiroshima 730 JAPAN

Department of Mathematics, College of General Education, Ibaraki University, Mrto 310, JAPAN 